\title{
CERTIFICACION PEFC DE MIELES BAJO GESTIÓN FORESTAL SOSTENIBLE
}

\author{
Rojas, Patricio ${ }^{6}$; Vidal, Rodrigo ${ }^{7}$; Molina, María Paz; \\ Rodríguez, Francisco" ${ }^{9}$ Espejo, Jaime ${ }^{10}$ y Gutiérrez, David ${ }^{11}$.
}

\section{RESUMEN}

En Chile existen alrededor de 10 mil explotaciones que administran más de 454 mil colmenas, las cuales generan una variada gama de productos apícolas como parte de la cadena productiva. La producción de miel, originada principalmente en especies melíferas del bosque nativo chileno, es exportada en cerca de un $90 \%$ a los mercados de la Unión Europea y Estados Unidos, constituyéndose en el principal producto pecuario primario exportado por Chile. La diferenciación de las mieles chilenas en mercados internacionales se produce por la composición del origen botánico, su fraccionamiento, certificación y valor agregado, como también por sus propiedades alimentarias y nutracéuticas.

Durante los meses de agosto de 2019 a mayo de 2020 se desarrolló un Proyecto Piloto con el objetivo de certificar la producción de mieles con el estándar de Cadena de Custodia PEFC Chile en patrimonio de las empresas Forestal Mininco SA y Forestal Arauco SA certificadas bajo el estándar de Gestión Forestal Sostenible CERTFOR/PEFC con apicultores de las comunas de Mulchén, Huépil, Cañete y Los Álamos. Esto con el objetivo adicional de dar mayor valor agregado a una producción sostenible de mieles para su comercialización en el mercado local e internacional de los apicultores de la Región del Bio Bio y también incorporar las plantaciones de Eucalyptus spp como posible insumo alimentario de los apiarios, dada la magnitud de este recurso forestal del país estimada en unas 860 mil hectáreas (INFOR, 2019).

Las empresas forestales otorgaron facilidades de acceso a los apicultores, a predios definidos de manera conjunta, para el establecimiento de los apiarios en sectores con plantaciones forestales, núcleos de bosque nativo y áreas en proceso de restauración, donde las abejas no solo se alimentaron y desarrollaron prósperamente sino que aumentaron su producción de miel por hasta llegar a 40kg/colmena. La Corporación CertforChile a través de su Sistema Chileno de Gestión Forestal Sostenible CERTFOR (PEFC Chile) se comprometió a coordinar el proceso de auditoría entre el Organismo de Certificación y los Apicultores bajo el Estándar de Cadena de Custodia PEFC y producir mieles certificadas en bosques gestionados de manera sostenible y capacitar y asesorar a los apicultores en la etapa de implementación del estándar. Financió los honorarios del Organismo de Certificación el primer año de auditoría y eximirá del pago de la cuota anual por uso de las marcas PEFC Chile, por el ciclo de certificación de 5 años. Los apicultores pertenecen a la región del Bio Bio y con importante experiencia en la producción apícola se comprometieron al pago de los honorarios del Organismo de Certificación a partir del segundo año, además de cumplir con toda la documentación asociada a la implementación del Estándar de Cadena de Custodia PEFC y participar en el proceso de auditoría para certificar su producción de miel en bosques certificados CERTFOR. INFOR se comprometió a efectuar el análisis de las muestras de mieles obtenidas del Piloto, según la Norma NCh 29812005 para certificar su

\footnotetext{
${ }_{7}^{6}$ INFOR. Sede Metropolitana. parojas@infor.cl

${ }^{7}$ PEFC CHILE. rodrigo.vidal@pefc.cl

${ }^{8}$ INFOR, Sede Bio Bio.mmolina@infor.cl

${ }^{9}$ Forestal Mininco. francisco.rodriguez@cmpc.cl

${ }_{11}^{10}$ Consultor. jespejoc@uc.cl

${ }^{11}$ Forestal Arauco. David.Gutierrez@arauco.com
} 
composición botánica, mediante el análisis meliso palinológico. Seis apicultores de Mulchén, Huépil, Cañete y Los Álamos finalmente certificaron la producción de sus mieles bajo el estándar de Cadena de Custodia PEFC. En este artículo se describe todo el proceso de certificación efectuado bajo los estándares de PEFC Chile, la participación de empresas, INFOR y los apicultores del Bio Bio de la primera certificación de la miel como PFNM en Chile.

Palabras clave: Certificación PEFC de mieles, Apicultores región del Bio Bio

\section{SUMMARY}

In Chile, there are around 10 thousand farms that manage more than 454 thousand hives, which generate a varied range of bee products as part of the production chain. Honey production, originating mainly from honey species from the Chilean native forest, is exported in about $90 \%$ to the markets of the European Union and the United States, becoming the main primary livestock product exported by Chile. The differentiation of Chilean honeys in international markets is produced by the composition of the botanical origin, its fractionation, certification and added value, as well as its food and nutraceutical properties. During the months of October 2019 and April 2020, a Pilot Project was developed with the objective of certifying the production of honeys under sustainable forest management PEFC Chile in the heritage of the companies Forestal Mininco and Forestal Arauco with beekeepers from the communes of Mulchén, Huepil, Cañete and Los Alamos. This with the additional objective of giving greater added value to the production of honeys for their commercialization in the local and international market of beekeepers in the Region of Bio Bio and also incorporating the Eucalyptus spp plantations as food for apiaries.

Forest companies permit access facilities to beekeepers, to jointly defined properties, for the establishment of apiaries in sectors with forest plantations, nuclei of native forest and areas undergoing restoration, where bees not only fed and developed prosperously but also increased their honey production.PEFC Chile undertook to coordinate the audit process between the Certification Body and Beekeepers under the Chain of Custody Standard and to produce certified honeys from sustainably managed forests, to train and advise beekeepers at the standard implementation stage; coordinate the audit process between the Certification Body and the beekeepers. It financed the Certification Body fees the first year of the audit and will exempt the annual fee for use of the PEFC Chile brands, for the 5-year cycle.

The beekeepers paid the Certification fees for the first year of the audit and subsidized the payment of the annual fee to the PEFC Chile Corporation for use of the PEFC brand or logo for a 5year cycle. They also promised to pay the Certification Body fees from the second year. The beekeepers belong to the Bio Bio Region, preferably selected for being part of the National Beekeeping Network and with significant experience in beekeeping production and the Official Animal Traceability Program of the SAG. The beekeepers promised to comply with all the documentation associated with the implementation of the PEFC Chile Chain of Custody Standard and to participate in the audit process to certify their honey production in certified forests. INFOR undertook to carry out in the laboratory the analysis of the honey samples obtained from the Pilot, according to Standard NCh 29812005 to certify their botanical composition, through melisopalinological analysis. Six beekeepers from Mulchén, Huepil, Cañete and Los Alamos certified the production of their honeys under sustainable forest management. This publication analyzes the entire certification process carried out under the PEFC Chile standards, the participation of companies, INFOR and the Bio Bio beekeepers of the first certification of honey as NWFP in Chile.

Key Words: PEFC honey certification, Bio Bio Region beekeepers. 


\section{INTRODUCCIÓN}

En Chile existen alrededor de 10 mil explotaciones que administran más de 454 mil colmenas, las cuales generan una variada gama de productos apícolas en la cadena productiva, siendo la producción nacional de 7 a 11 mil toneladas por año, de la cual $70 \%$ se exporta en tambores de $300 \mathrm{~kg}$. El consumo nacional corresponde al 30\% de la producción.

La producción de miel, originada principalmente en especies melíferas del bosque nativo chileno, es exportada en cerca de un $90 \%$ a los mercados de la Unión Europea y Estados Unidos, constituyéndose en el principal producto pecuario primario exportado por Chile.

La diferenciación de las mieles chilenas en mercados internacionales se produce por la composición del origen botánico según la Norma Chilena NCh2981. Of2005, su fraccionamiento, certificación y valor agregado, como también de sus propiedades alimentarias y nutracéuticas. Según ODEPA las tendencias de los principales exportadores van a la comercialización de altos volúmenes de precios muy por debajo del promedio, y la consolidación de las apuestas dirigidas a mieles de alta calidad y volúmenes que permitieron altos precios unitarios. (Barrera, 2018).

Los consumidores a nivel mundial se preocupan cada vez más por la seguridad alimentaria, incluidas las posibles impurezas en la miel asiática o de las falsificaciones. Los consumidores creen que comer productos locales de miel certificada podría ser una forma de evitar riesgos para la seguridad alimentaria.

Esta seguridad está garantizada por marcas de certificación que crean confianza en los consumidores y ayudan a sus decisiones en la compra. La triangulación y falsificación de miel corresponden a los principales problemas del comercio internacional de este alimento, ya que generan mayores costos correspondientes a la verificación de la calidad y origen de las mieles.

En el caso de Hungría el consumo de miel de origen local es muy importante y prefieren comprar estos productos directa o indirectamente de los apicultores. Al respecto los consumidores están bien informados en términos de certificación de la miel. Las marcas consideran en su fraccionamiento algunas características de la miel (región de origen, marca, nombre del productor, marcas de certificación).

Existe conciencia de las marcas de certificación las cuales ayudan a las decisiones del consumidor que están informados si estas se vieron afectadas por las falsificaciones de miel (Titanilla Oravecz, 2020).

INFOR participa como miembro de la Sub Comisión de Sustentabilidad y Territorio de la Comisión Apícola Nacional del Ministerio de Agricultura (INFOR-ODEPA, 2018), cuyo objetivo es fomentar el desarrollo sostenible de la apicultura y su relación con otros rubros, de forma de asegurar la sustentabilidad de la apicultura con la oferta de polen y néctar de masas boscosas.

INFOR además desarrolla un proyecto FNDR en la región del Bio Bio (INFOR, 2020) para el establecimiento de huertos melíferos, cuyo objetivo es la difusión de tecnologías silvícolas entre los apicultores para aumentar la producción y la calidad de las mieles diferenciadas ${ }^{12}$.

PEFC Chile ha participado en el primer proceso de certificación de mieles mono florales

\footnotetext{
12 Se entenderá por miel diferenciada aquella distinta a la miel a granel por efectos del origen botánico, su fraccionamiento y/o propiedades nutracéuticas.
} 
de Eucalyptus bajo gestión forestal sostenible en Uruguay, bajo el Estándar de PEFC Uruguay y este proyecto piloto verá la factibilidad de su implementación en Chile, para certificar el primer Producto Forestal No Maderero bajo este atributo sostenible.

Existen también experiencias de la relación de apicultores y las plantaciones de Eucalyptus en Brasil y Sudáfrica para la producción de mieles monoflorales (Barth, 1990).

Los apicultores se han manifestado interesados en producir mieles certificadas PEFC para su comercialización como mieles diferenciadas y fraccionadas tanto en el mercado nacional como internacional. Aunque no existen registros de publicaciones en Chile sobre la producción de mieles monoflorales de Eucalyptus estas son mieles importantes y comercializadas en Europa y Australia (Rodriguez, M., 2014).

Las plantaciones de Eucalyptus globulus y Eucalyptus nitens, con 588.543 y 270.076 ha, respectivamente (INFOR, 2019), representan las especies exóticas de más rápido crecimiento y mayor interés industrial para la producción de celulosa Kraft en Chile.

Este importante recurso floral y de néctar de las plantaciones constituye una potencial fuente para la producción de mieles monoflorales de Eucalyptus que no ha sido utilizada en el país para el fraccionamiento y diferenciación de las mieles.

En Europa, las principales áreas de producción de este tipo de miel se encuentran en Italia, España y Portugal, donde se producen mieles monoflorales de Eucalyptus globulus y Eucalyptus camaldulensis. Fuera de Europa, grandes cantidades de miel de eucalipto se producen en Australia donde el género es nativo y donde existen más de 800 especies.

Esto ha motivado el estudio de este tipo de miel por muchos autores: algunos de ellos melisopalinológico (Ricciardelli D’Albore y Vorwohl, 1979; Louveaux y Abed, 1984) y otros relacionados con el análisis físico-químicos (Accorti, 1995); Serra Bonvehı, 1990) y recientemente autores han estudiado los flavonoides (Ciappini, 2013).

Los estudios fisicoquímicos se basan en criterios como el contenido de azúcares, la conductividad eléctrica y el análisis de $\mathrm{pH}$, para complementar el análisis melisopalinológico, y actúan como los criterios principales para la caracterización de mieles monoflorales.

\section{OBJETIVOS}

Desarrollar una experiencia piloto con apicultores de las comunas de Mulchén, Huépil, Cañete y Los Álamos para establecer colmenas, tanto en plantaciones de Eucalyptus como en áreas de restauración ecológica de las empresas Forestal Mininco y Forestal Arauco, con el objetivo de certificar toda la cadena de trazabilidad de la producción de mieles para su certificación bajo el sistema PEFC, como mieles sostenibles producidas en bosques bajo gestión forestal sostenible CERTFOR/PEFC y obtener una certificación con alcance internacional.

Revisar la factibilidad de incorporar las plantaciones de Eucalyptus spp como posible insumo alimentario de los apiarios dada la magnitud de este recurso forestal del país 


\section{METODOLOGÍA}

\section{PEFC $^{13}$ Chile. Programa para la Homologación. Sistemas de Certificación Forestal}

La certificación forestal es un mecanismo de mercado cuyo objetivo es garantizar a los consumidores de productos forestales (madera, papel, paneles, otros) que dicho producto o servicio proviene de bosques cuya gestión se ha realizado bajo ciertos estándares, definidos por cada uno de los sistemas de certificación. Este proceso, cada vez con mayor intensidad, se ha transformado en un elemento diferenciador que aumenta la competitividad de los productos forestales en los mercados internacionales.

La certificación forestal consta de dos etapas, la primera es la Gestión Forestal Sostenible (GFS), que busca una gestión responsable de los bosques, bajo estrictos requerimientos de sostenibilidad $\mathrm{y}$, la segunda, garantizar la trazabilidad de los productos elaborados con materias primas que provienen de estos bosques sostenibles hasta el consumidor final, denominada Cadena de Custodia (CdC). El principal objetivo de una Cadena de Custodia $(\mathrm{CdC})$ es garantizar una conexión entre el ingreso del material certificado a un proceso y los productos elaborados, manteniendo el atributo "sostenible" de estos productos que provienen del bosque.

\section{La Experiencia de Uruguay}

Cada año, Forestal Oriental realiza un llamado nacional dirigido a apicultores locales para que hagan uso de las plantaciones de Eucalyptus para la producción de miel como producto complementario a la actividad forestal sostenible. Actualmente, 220 apicultores trabajan en plantaciones de Forestal Oriental del Uruguay. En 2017, estos apicultores tuvieron la oportunidad de participar en el comienzo de un plan piloto dirigido a aquellos interesados en certificar su producción de miel con sello PEFC. Como parte del proyecto, PEFC, un organismo europeo de certificación forestal que forma parte de una alianza global presente en 50 países incluyendo Uruguay, ha estado trabajando junto a UPM Forestal Oriental y organizaciones de regulación nacional.

La principal ventaja para el apicultor al obtener la certificación PEFC es la valorización de su producción de miel, a través del uso del logo PEFC en sus productos, reconocido en más de 70 países y el cual implica un origen forestal sostenible. Este sello permite una mayor apertura de mercado para la miel de origen forestal sostenible. "La certificación valida que se ha cumplido con todas las condiciones de trabajo y de higiene para mantener la calidad de esa miel, que es pura y no está mezclada con otras, y que en el predio donde se produce no hay presencia de productos químicos".

La certificación PEFC contempla dos aspectos fundamentales. En primer lugar, el manejo forestal de los bosques, que implica cómo se manejan estos para la producción, cuidando el medio

\section{3}

PEFC nace en Europa en el año 1999. Organización Gubernamental sin fines de lucro que se basa en el reconocimiento de sistemas nacionales de certificación y crea normas globales de gestión forestal sostenible, que sirvan como guía base para los sistemas de certificación forestal. PEFC promueve la gestión sostenible de los bosques y su industria derivada alrededor del mundo, a través de relaciones estratégicas globales, diálogos internacionales de partes interesadas y normas de certificación. Participa activamente en los espacios globales de decisión, debate y diálogo sobre el manejo sostenible de los bosques y su impacto en la sociedad y el medio ambiente a nivel mundial. PEFC en Chile está presente desde el año 2004, fecha en que se reconoce y se homologa el sistema chileno de certificación forestal (CERTFOR), hoy llamado PEFC Chile, el cual promueve localmente la certificación de GFS y CdC. 
ambiente, atendiendo a la legislación y a las relaciones sociales con la comunidad y con otras empresas. En segundo lugar, la certificación de cadena de custodia que certifica los procesos y la trazabilidad de cada producto que sale de un bosque certificado. Se certifica paso a paso, desde el producto inicial hasta el producto final. Esto significa que la miel producida por estos productores apícolas será certificada como sostenible en todo su proceso, lo cual es importante no solo para su comercialización, sino también para la salud de las abejas y el cuidado del medio ambiente.

\section{Requisitos de la Certificación PEFC para la Producción de Mieles Bajo Gestión Forestal Sostenible}

\section{- De los Apicultores}

Los apicultores incluidos en este proceso de certificación fueron preferentemente con importante experiencia en la producción apícola, por lo anterior se analizó su producción anterior, si disponía de equipamiento e implementos para la instalación, manejo, cosechas (centrifugas) y producción de las mieles en forma regular.

Los apicultores seleccionados fueron parte del Programa Oficial de Trazabilidad Animal ${ }^{14}$. En el caso de un productor de Arauco se consideró la inclusión de colmenas de media alza de forma de capturar la producción de mieles de la corta temporada de la floración de Eucalyptus (septiembre a diciembre).

Al final de la temporada los apicultores se comprometieron a informar la cantidad de miel cosechada por colmena y por el apiario en la temporada del proyecto piloto y entregar una muestra con una de respaldo para el análisis de laboratorio. Originalmente se consideraron cinco apicultores de la provincia del Bio Bio y cinco de Arauco, pero finalmente certificaron seis.

Las ventajas de obtener la certificación de Cadena de Custodia PEFC para la miel permiten valorizar el producto, demostrar el manejo responsable de la cadena de suministros, acceso a nuevos mercados, liderazgo en temas de sustentabilidad, cumplimiento con la legislación, trazabilidad y uso del sello PEFC ${ }^{15}$.

Los apicultores se comprometieron a cumplir con toda la documentación asociada a la implementación del Estándar de Cadena de Custodia PEFC y participar en el proceso de auditoría para certificar su producción de miel en bosques certificados CERTFOR/PEFC. Además, se consideró un aspecto de gran importancia para una buena producción apícola y que tiene relación con la sanidad de las colmenas.

Antes de ingresar las colmenas a los rodales seleccionados, se recomendó hacer un muestreo aleatorio del $10 \%$ de las colmenas. El análisis sanitario de dichas muestras debía arrojar resultados cualitativos y cuantitativos de Varroasis, Nosemosis y Acariosis. En caso de resultar positiva a una o a todas las parasitosis, se tomaron acciones tendientes a resolver los problemas sanitaros detectados para asegurar una buena producción de miel.

\footnotetext{
14

Desde el 01 de enero de 2005, el SAG cuenta con el Programa Oficial de Trazabilidad Animal, de gestión pública privada, que constituye una herramienta de apoyo a los programas oficiales del SAG en ámbitos de inocuidad de productos pecuarios, sanidad animal y certificación de exportaciones, garantizando la seguridad sanitaria de los productos pecuarios nacionales.

${ }_{15} \mathrm{https}$ ://www.upm.uy/prensa/todas-las-noticias/2019/04/upm-impulsa-primera-experiencia-mundial-encertificacion-apicola-con-origen-forestal-sostenible/
} 


\section{Cuadro $\mathrm{N}^{\circ} 1$}

\section{ANTECEDENTES DE LOS APICULTORES CERTIFICADOS POR PEFC CHILE}

\begin{tabular}{|c|c|c|c|c|c|c|}
\hline & \multicolumn{2}{|c|}{ Arauco } & \multicolumn{4}{|c|}{ Bio Bio } \\
\hline \multirow{2}{*}{$\begin{array}{c}\text { Antecedentes } \\
\text { personales }\end{array}$} & $\begin{array}{l}\text { Lorenzo } \\
\text { Flores }\end{array}$ & $\begin{array}{c}\text { Marcelo } \\
\text { Rodriguez }\end{array}$ & $\begin{array}{c}\text { Sonia } \\
\text { Mosquera }\end{array}$ & $\begin{array}{c}\text { Luis } \\
\text { Vasquez }\end{array}$ & $\begin{array}{c}\text { Jonny } \\
\text { Marquez }\end{array}$ & $\begin{array}{c}\text { Andres } \\
\text { Sanhueza }\end{array}$ \\
\hline & $13.808 .982-7$ & $10.966 .055-8$ & $11.017 .270-2$ & 8.698.011-8 & $16.498 .054-5$ & $17.076 .669-5$ \\
\hline Predio / localidad & $\begin{array}{l}\text { Caramavida } \\
\text { Seccion } 2 \\
\text { Los Álamos }\end{array}$ & $\begin{array}{l}\text { Cerro Alto } \\
\text { Los Álamos }\end{array}$ & Huepil & Huepil & Muchen & Mulchen \\
\hline $\begin{array}{l}\text { Cantidad de } \\
\text { apiarios / número } \\
\text { de colmenas }\end{array}$ & $\begin{array}{l}5 \text { apiarios } \\
500 \text { colmenas }\end{array}$ & $\begin{array}{l}1 \text { apiarios } \\
30 \text { colmenas }\end{array}$ & $\begin{array}{l}400 \\
\text { colmenas }\end{array}$ & 83 colmenas & 250 colmenas & $\begin{array}{c}400 \\
\text { colmenas }\end{array}$ \\
\hline $\begin{array}{l}\text { Rendimiento (kg } \\
\text { de miel/colmena) }\end{array}$ & 27 & 7,1 & & & & \\
\hline $\begin{array}{l}\text { Mercado } \\
\text { nacional o } \\
\text { internacional }\end{array}$ & Nacional & Nacional & Nacional & Nacional & $\begin{array}{c}\text { Nacional e } \\
\text { internacional }\end{array}$ & Nacional \\
\hline \multirow{2}{*}{$\begin{array}{l}\text { Produccion de } \\
\text { miel granel y/o } \\
\text { fraccionada }(\mathrm{kg})\end{array}$} & \multirow{2}{*}{$\begin{array}{c}30 \% \\
\text { fraccionado } \\
70 \% \text { granel }\end{array}$} & \multirow{2}{*}{$\begin{array}{c}82 \% \\
\text { fraccionado } \\
8 \% \text { granel }\end{array}$} & 7.800 & 1.700 & 10.000 & 16.500 \\
\hline & & & & & & \\
\hline \multirow{2}{*}{$\begin{array}{l}\text { Precios / kg } \\
\text { nacional o } \\
\text { internacional }\end{array}$} & $\begin{array}{c}\$ 1.700 \mathrm{~kg} \\
\text { granel }\end{array}$ & & $\$ 1.900$ & $\$ 1.800$ & $\$ 1.700$ & $\$ 1.650$ \\
\hline & $\begin{array}{c}\$ 8.400 \mathrm{~kg} \\
\text { fraccionado }\end{array}$ & $\begin{array}{c}\$ 8.400 \mathrm{~kg} \\
\text { fraccionado }\end{array}$ & & & & \\
\hline
\end{tabular}

\section{- De las Empresas Forestales (Forestal Mininco SA y Forestal Arauco SA)}

Las empresas forestales buscan proporcionar un refugio ecosistémico para las abejas y promover la apicultura de manera sostenible y colaborativa con las comunidades vecinas. Contribuir al mantenimiento del rubro es un aporte a la protección del patrimonio medio ambiental y social del país, así como una apuesta por repensar un futuro sostenible donde los productos melíferos y sus derivados participen más activamente de las cadenas de valor comercial y social. La Apicultura aporta con la seguridad y soberanía alimentaria, alienta el turismo rural y se posiciona como un área estratégica para la conservación de especies nativas, lo que mitiga el cambio climático. Las abejas prestan un servicio ecosistémico incalculable e imprescindible para la biodiversidad genética a través del flujo génico mediante la polinización. A su vez constituyen un patrimonio social concreto desde las diversas expresiones de desarrollo local.

Este proyecto piloto permite generar relaciones de colaboración con diversas partes interesadas que contribuyen a incrementar sosteniblemente los usos sociales, económicos y culturales del territorio donde se está trabajando. Sumado a este objetivo primordial, se crea y difunde conocimiento de manera asociativa, estableciendo mecanismos de divulgación que contribuyen a generar iniciativas similares. Desde el punto de vista ecosistémico, el proyecto hace sinergia con los esfuerzos de restauración que llevan estas empresas, mejorando sustancialmente la polinización de diversas especies nativas de los bosques colindantes que proveen de semillas a zonas en proceso de restauración. 
Por este motivo, este vínculo de trabajo puede ser considerado como una mejora en la provisión de servicios ecosistémicos, asociados a la biodiversidad nativa, asegurando la sostenibilidad de los esfuerzos de restauración nacional buscando armonizar los aspectos sociales, ambientales y económicos. Para dar inicio al proyecto piloto Forestal Arauco y Forestal Mininco otorgaron facilidades de acceso a los apicultores a los predios definidos previamente. La selección de los sitios busca asegurar la alimentación natural de las colmenas (polen, néctar, agua) y su seguridad. Para mayor precisión de los lugares se adjuntan los planos de ubicación de los lugares autorizados.

\section{Selección de los Rodales y Áreas de Restauración Ecológica}

El propósito en los rodales de Eucalyptus globulus fue aprovechar la próxima floración de la especie (agosto a diciembre, con un peak de floración entre septiembre - octubre), en el caso de de Eucalyptus nitens en la Hacienda Rucamanqui la ventana de floración corresponde entre Julio y Enero. Para el caso de Eucalyptus viminalis la floración ocurre entre diciembre y marzo por lo cual se establecieron colmenas hasta completar la floración. De preferencia se prospectaron rodales con mayor espaciamiento ( $3 \times 3$ metros, 1.100 árb/ha y preferentemente con yemas florales en preántesis) para lo cual se hizo una prospección preliminar del avance de la floración en todos los sitios). El rodal debía ser mayor a 8 años, en edad reproductiva y con gran producción de flores (se pudo estimar con la producción de botones o yemas florales, en pre ántesis). Debido a que la floración es menor al interior del rodal, las colmenas se establecieron en los bordes de la plantación, de forma que las abejas podían pecorear los árboles orilleros que son los que tienen mayor floración.
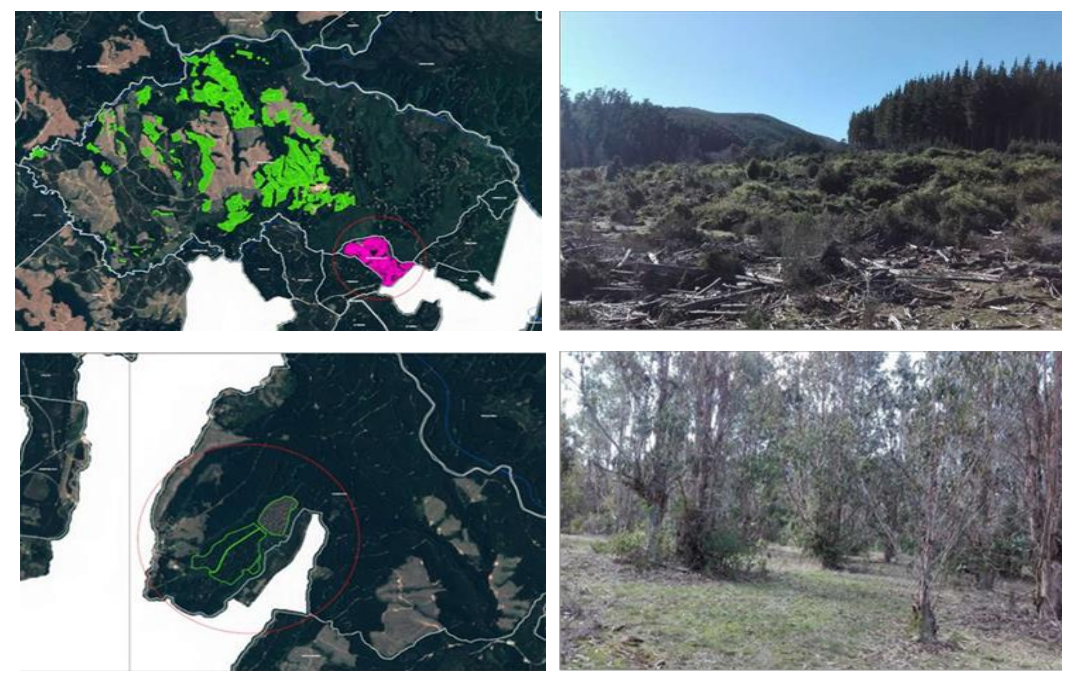

Figura $\mathrm{N}^{\circ} 1$

ÁREA DE RESTAURACION ECOLÓGICA LOS CORRALES (ARRIBA)

HUERTO SEMILLERO CLONAL DE Eucalyptus globulus. CERRO ALTO (ABAJO). FORESTAL ARAUCO 
En el caso del Huerto Semillero Clonal de Cerro Alto, debido al amplio espaciamiento del rodal fue posible establecer las colmenas al centro de la plantación. Para este efecto, en cada predio cada apicultor estableció 30 colmenas en las plantaciones cercanas a su predio y también se incluyeron otras 30 colmenas como control de actividad normal de productor para comparar al final de la temporada la composición de las mieles y la cantidad de miel producida por colmena y con apiario. El eucalipto se clasifica en el grupo de las especies que contribuyen al crecimiento y desarrollo de las colmenas, pues su floración se produce a comienzos de primavera (septiembreoctubre). Para hacer coincidir el peack de la floración del eucaliptos con el peack de máximo desarrollo de la colmena, en términos de población de abejas, se proporcionó alimentación de estímulo a las colmenas al menos 40 días (6 semanas) antes de la máxima floración, esto con la finalidad de tener la mayor cantidad posible de abejas pecoreadoras que permitan la generación de excedentes en cuanto a la producción de miel. Dicha alimentación de estímulo corresponde a jarabe de fructosa 1:1 complementado con $5 \mathrm{ml}$ de vitamina liquida por litro de jarabe.
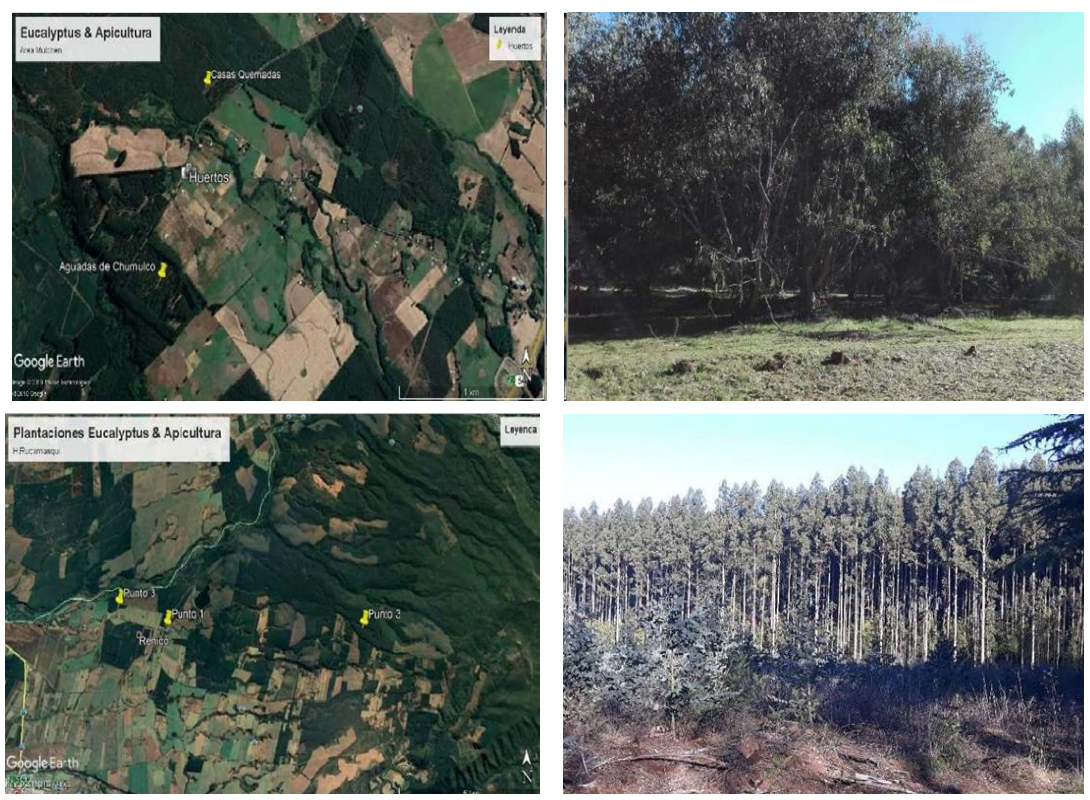

Figura $\mathbf{N}^{\circ} 2$

HUERTO SEMILLERO CLONAL DE Eucalyptus globulus. CHUMULCO (ARRIBA)

PLANTACIONES DE Eucalyptus nitens EN HACIENDA RUCAMANQUI. FORESTAL MININCO (ABAJO).

\section{Rol de PEFC Chile}

Coordinar el proceso de certificación de los apicultores bajo el Estándar de Cadena de Custodia PEFC para producir mieles certificadas de bosques gestionados en forma sostenible. 
Capacitar y asesorar a los apicultores en la etapa de implementación del Estándar.

Coordinar el proceso de auditoría entre el Organismo de Certificación y los apicultores.

Costear los honorarios del Organismo de Certificación el primer año de auditoría.

Eximir del pago de la primera cuota anual a la Corporación CertforChile por uso de la marca PEFC, por un ciclo de 5 años.

\section{Rol de INFOR}

Compromiso de efectuar en laboratorio el análisis de las muestras de mieles obtenidas del Piloto, según la Norma NCh 29812005 para certificar su composición botánica, mediante el análisis melisopalinológico. Estos análisis serán financiados por INFOR a través de su proyecto de Flora Melífera (FNDR del Bio Bio)

\section{PROCESO DE CERTIFICACIÓN}

- Reuniones Informativas: Se realizaron diferentes reuniones informativas con los apicultores, en ellas se les entregó información básica del proyecto, de la forma de trabajo y requisitos necesarios para poder participar en él. Estas reuniones se realizaron con los grupos antes mencionados y contaron con la presencia de representantes de las empresas participantes (Forestal Arauco SA y Forestal Mininco SA), INFOR y PEFC Chile.

- Diagnóstico: El proceso de diagnóstico se realizó en forma individual con cada uno de los apicultores en sus respectivas instalaciones, en esta etapa se evaluó la forma de trabajo y se establecieron las brechas y plan de trabajo para cumplir con los requisitos para lograr el objetivo de certificarse bajo el Estándar de CdC PEFC. Para realizar el diagnóstico se utilizó una "Matriz de Diagnóstico del Estándar de CdC PEFC" la cual consiste en evaluar el cumplimiento de cada uno de los requisitos de la norma, considerando la documentación que les permite cómo cumplir con estos requisitos, y de ser necesario, las medidas o acciones se deben realizar para su cumplimiento.

- Implementación de la documentación: En las instalaciones de los apicultores se realizaron diferentes reuniones, para establecer en detalle los procesos asociados a la producción de miel y de certificación de CdC. En este contexto, cada uno de los apicultores (con el apoyo de PEFC Chile) elaboró un "Manual de CdC" , el que consiste en un documento general, donde se describe su forma de trabajo y cómo se dará cumplimiento a cada uno de los requisitos del estándar, considerando los temas principales.

- Descripción del proceso trazable: Consiste en describir la forma en que los apicultores demuestran que mantienen el control de todos sus materiales y productos de modo que sus productos certificados no se mezclen con productos sin certificación, entre otros puntos está la identificación de sus apiarios y el lugar de instalación de estos, de modo de demostrar que se encuentran en patrimonios certificados con GFS CERTFOR/PEFC, y que los productos obtenidos de estos no se mezclan con el producto de otros apiarios que no se encuentran dentro de patrimonios certificados. 
- Definición de Responsabilidades: En este punto se identifica quien asumirá la responsabilidad del control de materiales y los compromisos que se adquieren con la administración del proceso de certificación.

- Venta de productos certificados: Se describe las obligaciones que deben asumir los apicultores al momento de vender sus productos certificados, esto corresponde a la entrega de facturas $u$ otros documentos de venta que incluirán la declaración formal de CdC y sus respectivos códigos de CdC.

- Capacitación: De modo de que los participantes obtuvieran mayor información y conocimientos del estándar de CdC PEFC se realizaron cursos de capacitación donde se incorporaron los requisitos del Estándar de CdC PEFC y la forma en que los apicultores deben cumplir. Al completar el curso los participantes obtuvieron un diploma de Auditor Interno de CdC PEFC, siendo este un registro y comprobante de los conocimientos adquiridos a través de capacitación.

- Tercerización: Algunos apicultores realizan parte de sus procesos en dependencias de externos, estos procesos son principalmente extracción y fraccionamiento. Dado lo anterior, se realizaron visitas a los procesos de tercerización y se entregaron las medidas que deben realizar para poder cumplir con el proceso de certificación. Estas medidas fueron no mezclar productos certificados PEFC con productos sin certificación, no tener mano de obra infantil y permitir el acceso para posibles auditorías internas y/o externas. Para oficializar este compromiso los representantes de estas salas firmaron una Auto declaración de Prestadores de Servicios.

- Auditoría Interna: Los apicultores (con el apoyo de PEFC Chile) realizaron sus auditorías internas, las que consisten en evaluar el estado de cumplimiento de sus procesos con respecto al Estándar de CdC PEFC, de modo de detectar posibles hallazgos y corregirlos previo a la auditoria externa.

- Documentación asociada: Como parte del proceso de implementación se utilizaron y complementaron documentos o registros utilizados por los apicultores, listados a continuación:

- Registro de inscripción en el sistema de información pecuaria (SIPEC).

- Registro de inscripción en registro de apicultores de miel de exportación (RAMEX).

- Registro producción primaria de miel.

○ Registro sala de extracción.

- Registro resolución exenta sala de fraccionamiento del SEREMI de salud.

- Facturas de venta.

- Formulario de Registro de Apicultores y Declaración de Apiarios (F-PP-TZ-006)

- Registro de Ingreso y Uso de Medicamentos de Uso Veterinario en Apiarios RAMEX 

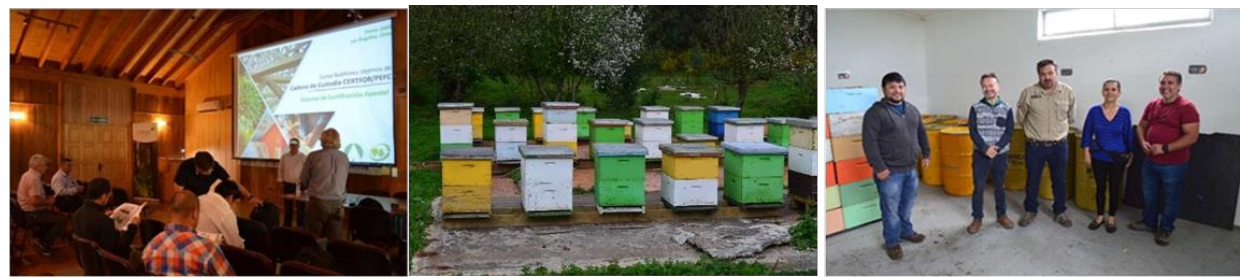

Figura $\mathrm{N}^{\circ} 3$

PROCESO DE CERTIFICACIÓN PEFC CHILE. CHARLA INFORMATIVA ESTABLECIMIENTO DE COLMENAS Y AUDITORÍA DE CADENA DE CUSTODIA

\section{ANÁLISIS MELISOPALINOLÓGICO DE LAS MIELES}

Las mieles producidas bajo la norma de certificación fueron enviadas al laboratorio de la Facultad de Agronomía de la Universidad Católica de Chile y analizadas bajo la norma Oficial NCh 2981.Of 2005 (Montenegro, 2008). Este análisis permite la exportación de las mieles con certificado de origen botánico y geográfico, evidenciando, al mismo tiempo, la variedad de la flora nativa utilizada por las abejas (Ramírez y Montenegro, 2004, 2000; Montenegro, 2003; Avila, 1993).
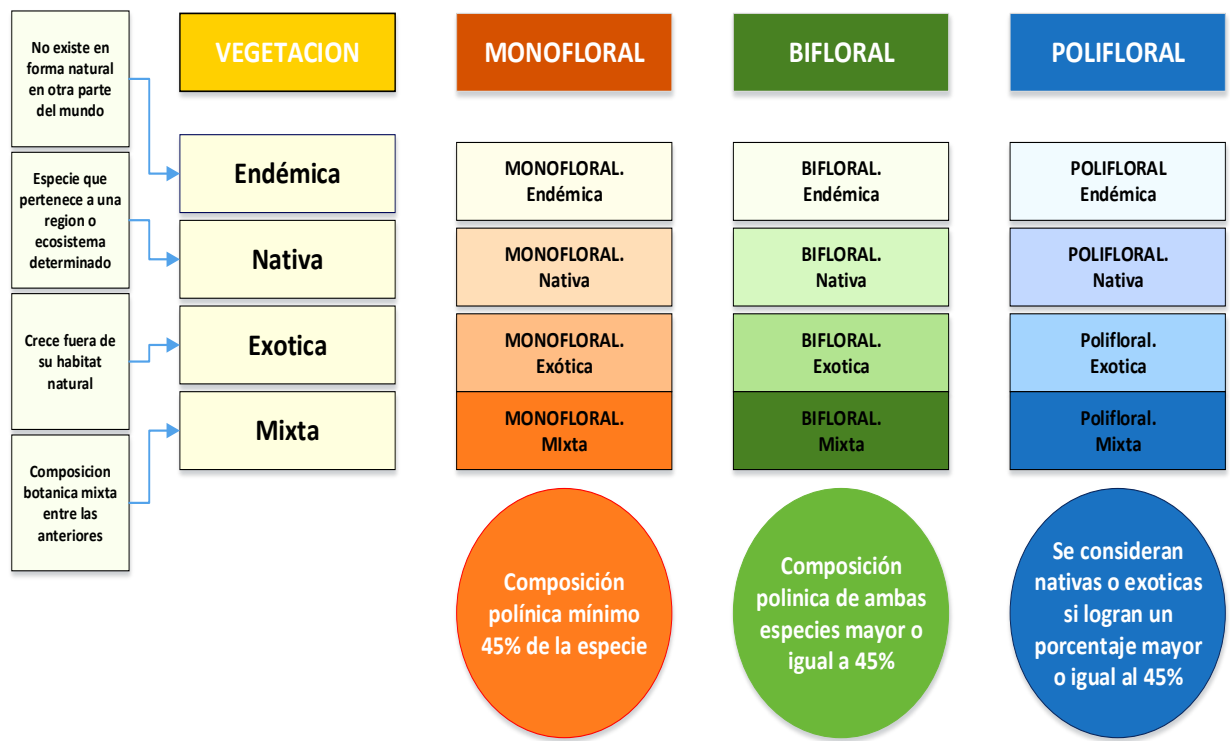

Figura $\mathrm{N}^{\circ} 4$

NORMA CHILENA OFICIAL NCh 2981 OF 2005

CARACTERIZACIÓN BOTÁNICA DE LAS MIELES POR ANÁLISIS MELISOPALINOLOGICO 
Esta norma permite la clasificación del origen botánico de las mieles según la flora acompañante de las colmenas en cuatro tipos: endémica, nativa, exótica y mixta. Esto es posible en cuanto la morfología de los granos de polen que es única para cada especie y permite su identificación y cuantificación en laboratorio.

\begin{tabular}{|c|c|c|c|c|c|c|}
\hline \multirow{4}{*}{$\begin{array}{c}\text { Apicultor. } \\
\text { Código Muestra }\end{array}$} & \multicolumn{5}{|c|}{$\begin{array}{c}\text { Cuadro } N^{\circ} 2 \\
\text { RESULTADOS DE LOS ANÁLISIS MELISOPALINOLÓGICOS }\end{array}$} & \\
\hline & \multicolumn{2}{|c|}{ Arauco } & \multicolumn{4}{|c|}{ Bio Bio } \\
\hline & $\begin{array}{l}\text { Lorenzo } \\
\text { Flores }\end{array}$ & $\begin{array}{c}\text { Marcelo } \\
\text { Rodriguez }\end{array}$ & $\begin{array}{c}\text { Sonia } \\
\text { Mosquera }\end{array}$ & $\begin{array}{c}\text { Luis } \\
\text { Vásquez }\end{array}$ & $\begin{array}{c}\text { Jonny } \\
\text { Marquez }\end{array}$ & $\begin{array}{c}\text { Andres } \\
\text { Sanhueza }\end{array}$ \\
\hline & M2023 & M2024 & M2025 & M2022 & M2026 & M2021 \\
\hline \multirow{2}{*}{$\begin{array}{l}\text { Ubicación } \\
\text { colmenas }\end{array}$} & Caramavida & Cerro Alto & \multirow{2}{*}{ Huepil } & \multirow{2}{*}{ Huepil } & \multirow{2}{*}{ Mulchen } & \multirow{2}{*}{ Mulchen } \\
\hline & Los Álamos & Los Álamos & & & & \\
\hline Origen botánico & $\begin{array}{l}\text { Monofloral } \\
\text { Tineo }\end{array}$ & $\begin{array}{c}\text { Polifloral } \\
\text { exotica }\end{array}$ & Polifloral & Polifloral & Polifloral & Polifloral \\
\hline \begin{tabular}{|l} 
Granos \\
polínicos de \\
Eucalyptus (\%)
\end{tabular} & 10,316 & 29,784 & 21,987 & 9,453 & 19,548 & 16,358 \\
\hline
\end{tabular}

La norma permite también diferenciar las mieles monoflorales, biflorales y poliflorales en cuanto a la fracción polínica obtenida, de esta forma las mieles con una fracción polínica de una especie superior al $45 \%$ son clasificadas como monoflorales, biflorales cuando la composición de ambas especies es mayor o igual al $45 \%$, o mezclas en diferentes proporciones como poliflorales, en este caso se consideran nativas o exóticas si logran un porcentaje superior al $45 \%$. Los resultados obtenidos con las mieles de los apicultores fueron 1 monofloral de tineo en el área de restauración y 5 poliforales en aquellas colmenas establecidas en plantaciones de eucaliptos, con distinta participación de fracción polínica de la especie, pero siempre presentes en rangos de 10 a $30 \%$.

\section{CONCLUSIONES}

Todas las mieles muestreadas tuvieron un componente de Eucalyptus (hasta un $33,4 \%$ en la muestra M2024), pero no se produjeron mieles monoflorales ( $>45 \%$ de los granos polínicos).

Todas las mieles muestreadas tuvieron un componente de alfalfa chilota, con una miel monofloral $(67,3 \% \%$ en la muestra M2022).

En las áreas de restauración se obtuvo una miel monofloral de tineo (muestra M2023) con un $51,2 \%$ de granos polínicos de la especie.

Los apicultores cumplieron con el programa Oficial de Trazabilidad Animal, la Cadena de Custodia (trazabilidad del proceso de producción de mieles) de la norma de PEFC Chile y la auditoría para su aprobación. Para la determinación del origen botánico sus mieles 
fueron analizadas bajo la Norma Chilena Oficial NCh 2981.Of 2005 en los laboratorios de la Facultad de Agronomía de la Universidad Católica de Chile.

El proceso de certificación PEFC Chile permite asegurar a los consumidores una miel original, sin riesgo de falsificación, aumentar la producción de sus colmenas y mejores sus precios en el mercado nacional e internacional asegurando que su producción se genera en bosques bajo manejo forestal sustentable, protegiendo la naturaleza y proporcionando un refugio sistémico de las colmenas.

Primer sello nacional PEFC de producción apícola con origen forestal sostenible.

\section{REFERENCIAS}

Accorti, M. 1995. Characterization of unifloral honey. Apidologie, 453-465.

Avila, 1993. La flora nativa sustentadora de colmenas de Apis mellifera en Pichidangui, cuarta region de Chile. Ciencia e Investigacion Agraria. v. 20(3) p. 119-125., 20(3) p. 119-125.

Barth, O. M., 1990. Pollen in Monofloral Honeys from Brazil. Journal of Apicultural Research, Vol (29) 89-94.

Barrera, D., 2018. Apicultura Chilena: Actualización de Mercado y Estadísticas Sectoriales. Oficina de Estudios y Políticas Agrarias.

Ciappini, M. 2013. El color como indicador de flavonoides en la miel. Revista Ciencia Tecnologia, 59-63.

INFOR, 2019. EI Sector Forestal Chileno 2019. Instituto Forestal, Chile, p 48

INFOR, 2020. INFOR apoya al sector apícola con plantación de 100 huertos melíferos en Bio Bio y Ñuble. En: https://www.infor.cl/index.php/noticias/554-infor-apoya-al-sector-apicola-con-plantacion-de-100-huertos-meliferosen-biobio-y-nuble

INFOR-ODEPA, 2018. Subcomisión Sustentabilidad y Territorio Comisión Nacional de Apicultura.

Louveaux, J. y Abed, L., 1984. Les Miels D'Afrique du NORD el leur Spectre Pollinique. Apidologie, Springer Verlag, 15 (2), pp.145-170. ffhal-00890621f

Montenegro, G. 2003. ORIGEN BOTANICO Y PROPIEDADES QUIMICAS DE LAS MIELES DE LAREGION MEDITERRANEAARIDADE CHIL. CIENCIAE INVESTIGACION AGRARIA, 162-173.

Montenegro, Gloria, 2008. Application of the Chilean Official Standard to Desígnate the Botanical Origins of Honey for the Characterization of the Apicultural Production. Ciencia e investigación Agraria, 35(2): 181-190.

Ricciardelli d'Albore, G., y Vorwohl, G., 1979. Tipos de Miel Monofloral de la Región del Mediterráneo, verificados mediante análisis microscópicos de la miel [plantas melíferas. Ciencia y Abejas (Argentina), v. 5(20) p. 5-6.. .

Rodriguez, M., 2014. Characterization of Eucalyptus globulus Honeys Produced in the Eurosiberian Area of the Iberian Peninsula. International Journal of Food Properties, 17:2177-2191.

Serra Bonvehi, J., 1990. Physico-chemical Properties. Composition and Pollen Spectrum of Eucalyptus Honey Produced in Spain. Anales de Bromatologia, 41(1) 41-56.

Titanilla Oravecz, L. M., 2020. Consumers Preferences for Locally Produced Honey in Hungary. Acta Universitatis Agriculturae et Silviculturae Mendelianae Brunensis, 407-416. 